\title{
Fixed-point theorems for nonlinear operators with singular perturbations and applications
}

\author{
Baoqiang Yan', Donal O'Regan ${ }^{2,3}$ and Ravi P Agarwal ${ }^{3,4^{*}}$
}

"Correspondence:

Agarwal@tamuk.edu

${ }^{3}$ Department of Mathematics, Faculty of Science, King Abdulaziz

University, Jeddah, Saudi Arabia ${ }^{4}$ Department of Mathematics, Texas A\&M University-Kingsville, Kingsville, Texas 78363, USA

Full list of author information is

available at the end of the article

\begin{abstract}
In this paper, using fixed-point index theory and approximation techniques, we consider the existence and multiplicity of fixed points of some nonlinear operators with singular perturbation. As an application we consider the existence and multiplicity of positive solutions of singular systems of multi-point boundary value problems, which improve the results in the literature.
\end{abstract}

Keywords: boundary value problems; singularity; fixed-point index

\section{Introduction}

In this paper we consider the problem

$$
x=A x+\lambda B x,
$$

where $A$ is continuous and compact and $B$ is a singular continuous and compact operator (defined in Section 2).

In the study of nonlinear phenomena many models give rise to singular boundary value problems (singular in the dependent variable) (see [1-3]). In [4], Taliaferro showed that the singular boundary value problem

$$
\left\{\begin{array}{l}
y^{\prime \prime}+q(t) y^{-\alpha}=0, \quad 0<t<1, \\
y(0)=0=y(1),
\end{array}\right.
$$

has a $C[0,1] \cap C^{1}(0,1)$ solution; here $\alpha>0, q \in C(0,1)$ with $q>0$ on $(0,1)$ and $\int_{0}^{1} t(1-$ t) $q(t) d t<\infty$. For more recent work we refer the reader to [5-14] and the references therein.

In this paper we consider abstract singular operators (defined in Section 2) and we consider the existence and multiplicity of fixed points of some nonlinear operators with singular perturbations. As an application we discuss the existence and multiplicity of positive solutions of singular systems of multi-point boundary value problems.

\section{Fixed-point theorems}

Let $E$ be a Banach space, $P$ a cone of $E, \Omega \subseteq E$ bounded and open. The following theorems are needed in our paper.

O2014 Yan et al.; licensee Springer. This is an Open Access article distributed under the terms of the Creative Commons Attribution License (http://creativecommons.org/licenses/by/2.0), which permits unrestricted use, distribution, and reproduction in any medium, provided the original work is properly cited. 
Theorem 2.1 ([8]) Suppose $\theta \in \Omega, A: P \cap \bar{\Omega} \rightarrow P$ is continuous and compact and

$$
A x \neq \mu x, \quad \forall x \in P \cap \partial \Omega, \mu \geq 1 .
$$

Then

$$
i(A, P \cap \Omega, P)=1 .
$$

Theorem 2.2 ([8]) Assume that $A: P \cap \bar{\Omega} \rightarrow P$ is continuous and compact. If there exists a compact and continuous operator $K: P \cap \partial \Omega \rightarrow P$ such that

(1) $\inf _{x \in P \cap \partial \Omega}\|K x\|>0$;

(2) $x-A x \neq \lambda K x, \forall x \in P \cap \partial \Omega, \lambda \geq 0$,

then

$$
i(A, P \cap \Omega, P)=0 .
$$

Now we give a new definition.

Definition 2.1 If $B: P-\{\theta\} \rightarrow P$ is continuous with

$$
\lim _{x \rightarrow \theta, x \in(P-\{\theta\})}\|B x\|=+\infty
$$

and $B(\{x \in P \mid r \leq\|x\| \leq R\})$ is relatively compact, for any $0<r<R<+\infty$, then $B: P-\{\theta\} \rightarrow$ $P$ is called a singular continuous and compact operator.

Remark Consider

$$
\begin{aligned}
& x^{\prime \prime}(t)+a(t) x^{-\gamma}(t)=0, \quad t \in(0,1), \\
& x(0)=0, \quad x(1)=0,
\end{aligned}
$$

where $1>\gamma>0$ and $a(t) \in C((0,1),(0,+\infty)) \cap L^{1}(0,1)$ or equivalently

$$
x(t)=\int_{0}^{1} G(t, s) a(s) x^{-\gamma}(s) d s, \quad t \in[0,1]
$$

where

$$
G(t, s)= \begin{cases}s(1-t), & 0 \leq s \leq t \leq 1 \\ t(1-s), & 0 \leq t \leq s \leq 1\end{cases}
$$

Set

$$
P:=\{x \in C[0,1]: x(t) \geq t(1-t)\|x\|\},
$$

where $\|x\|=\max _{t \in[0,1]}|x(t)|$. For $x \in P-\{\theta\}$, let

$$
(B x)(t):=\int_{0}^{1} G(t, s) a(s) x^{-\gamma}(s) d s, \quad t \in[0,1] .
$$


It is easy to see that $B: P-\{\theta\} \rightarrow P$ is a singular continuous and compact operator (see $[7,14])$.

Theorem 2.3 Suppose that $\theta \in \Omega, A: P \cap \bar{\Omega} \rightarrow P$ is continuous and compact and $B: P$ $\{\theta\} \rightarrow P$ is singular continuous and compact. Assume that

$$
A x \neq \mu x, \quad \forall x \in P \cap \partial \Omega, \mu \geq 1 .
$$

Then there exists a $\lambda_{*}>0$ such that, for any $\lambda \in\left(0, \lambda_{*}\right)$, there exist $x_{\lambda} \in P \cap \Omega-\{\theta\}$ with

$$
x_{\lambda}=A x_{\lambda}+\lambda B x_{\lambda} .
$$

Proof Choose $x_{0} \in P-\{\theta\}$, and define

$$
B_{n} x=B\left(x+\frac{1}{n} x_{0}\right), \quad \forall x \in P, n \in \mathbb{N} .
$$

Set

$$
\gamma:=\inf _{(\mu, x) \in[1,+\infty) \times P \cap \partial \Omega}\|\mu x-A x\| .
$$

Now we claim that

$$
\gamma>0 \text {. }
$$

If $\gamma=0$, there exists $\left\{\left(\mu_{n}, x_{n}\right)\right\} \subseteq[1,+\infty) \times P \cap \partial \Omega$ such that

$$
\lim _{n \rightarrow+\infty}\left\|\mu_{n} x_{n}-A x_{n}\right\|=0
$$

First, we show $\left\{\mu_{n}\right\}$ is bounded.

To see this suppose $\left\{\mu_{n}\right\}$ is unbounded. Without loss of generality, we assume that $\lim _{n \rightarrow+\infty} \mu_{n}=+\infty$. Then

$$
0 \leftarrow\left\|\mu_{n} x_{n}-A x_{n}\right\| \geq\left|\mu_{n}\right|\left\|x_{n}\right\|-\left\|A x_{n}\right\| \geq \mu_{n} \inf _{x \in P \cap \partial \Omega}\|x\|-\left\|A x_{n}\right\| \rightarrow+\infty,
$$

and this is a contradiction.

Next, we show that there exists a $\left(\mu_{0}, x_{0}\right) \in[1,+\infty) \times P \cap \partial \Omega$ such that

$$
\left\|\mu_{0} x_{0}-A x_{0}\right\|=0
$$

The boundedness of $\left\{\mu_{n}\right\}$ means that $\left\{\mu_{n}\right\}$ has a convergent subsequence. Without loss of generality, we assume that $\mu_{n} \rightarrow \mu_{0} \geq 1$. Since $\left\{x_{n}\right\}$ is bounded and $A$ is continuous and compact, $\left\{A x_{n}\right\}$ has a convergent subsequence $\left\{A x_{n_{i}}\right\}$ with $\lim _{n_{i} \rightarrow+\infty} A x_{n_{i}} \rightarrow y_{0}$. From (2.3), we have

$$
\lim _{n_{i} \rightarrow+\infty}\left\|\mu_{n_{i}} x_{n_{i}}-A x_{n_{i}}\right\|=0
$$


which implies that

$$
\mu_{n_{i}} x_{n_{i}} \rightarrow y_{0}, \quad n_{i} \rightarrow+\infty
$$

Then

$$
x_{n_{i}} \rightarrow \frac{1}{\mu_{0}} y_{0}, \quad \text { as } n_{i} \rightarrow+\infty .
$$

Let $x_{0}=\frac{1}{\mu_{0}} y_{0}$. Clearly, $x_{0} \in P \cap \partial \Omega$ and

$$
\left\|\mu_{0} x_{0}-A x_{0}\right\|=\lim _{n_{i} \rightarrow+\infty}\left\|\mu_{n_{i}} x_{n_{i}}-A x_{n_{i}}\right\|=0,
$$

which contradicts (2.1).

Let

$$
\beta_{n}=\sup _{x \in P \cap \partial \Omega}\left\|B_{n} x\right\| .
$$

Now we claim that

$$
\sup _{n \in \mathbb{N}} \beta_{n}<+\infty
$$

To see this suppose that

$$
\sup _{n \in \mathbb{N}} \beta_{n}=+\infty
$$

Without loss of generality, assume that

$$
\lim _{n \rightarrow+\infty} \beta_{n}=+\infty
$$

which implies that there exists a sequence $\left\{x_{n}\right\} \in P \cap \partial \Omega$ such that

$$
\lim _{n \rightarrow+\infty}\left\|B_{n} x_{n}\right\|=\lim _{n \rightarrow+\infty}\left\|B\left(x_{n}+\frac{1}{n} x_{0}\right)\right\|=+\infty .
$$

For all $x \in P \cap \partial \Omega$, we have

$$
\left\|x+\frac{1}{n} x_{0}\right\| \leq\|x\|+\left\|x_{0}\right\| \leq \sup _{x \in P \cap \Omega}\|x\|+\left\|x_{0}\right\|:=R<+\infty .
$$

Since, for any $r>0, B: P \cap\{x: r \leq\|x\| \leq R\}$ is relatively compact, (2.5) guarantees that there exists a subsequence $\left\{x_{n_{i}}\right\} \subseteq\left\{x_{n}\right\}$ such that

$$
\lim _{n_{i} \rightarrow+\infty}\left\|x_{n_{i}}+\frac{1}{n_{i}} x_{0}\right\|=0 .
$$

Thus

$$
\lim _{n_{i} \rightarrow+\infty}\left\|x_{n_{i}}\right\|=0,
$$

which implies that $\theta \in P \cap \partial \Omega$. This contradicts $\theta \in \Omega$. Hence, (2.4) holds. 
Set

$$
\beta:=\sup _{n \in \mathbb{N}} \beta_{n}<+\infty
$$

and

$$
\lambda_{*}:=\frac{\gamma}{\beta}>0
$$

For $0<\lambda<\lambda_{*}, x \in P \cap \partial \Omega, \mu \geq 1$, we have

$$
\left\|A x+\lambda B_{n} x-\mu x\right\| \geq\|A x-\mu x\|-\lambda\left\|B_{n} x\right\| \geq \gamma-\beta \lambda>0 .
$$

Theorem 2.1 guarantees that

$$
i\left(A+\lambda B_{n}, P \cap \Omega, P\right)=1 .
$$

Note (2.6) guarantees that, for any $\lambda \in\left(0, \lambda_{*}\right)$, there exists a $\left\{x_{n}\right\} \subseteq P \cap \Omega$ such that

$$
x_{n}=A x_{n}+\lambda B_{n} x_{n}, \quad n \in \mathbb{N} \text {. }
$$

Now we show that

$$
\inf _{n \in \mathbb{N}}\left\|x_{n}\right\|>0
$$

which implies that

$$
\inf _{n \in \mathbb{N}}\left\|x_{n}+\frac{1}{n} x_{0}\right\|>0
$$

To see this suppose that

$$
\inf _{n \in \mathbb{N}}\left\|x_{n}\right\|=0
$$

Then there exists a $\left\{x_{n_{i}}\right\}$ such that

$$
\lim _{n_{i} \rightarrow+\infty}\left\|x_{n_{i}}\right\|=0
$$

and so

$$
\lim _{n_{i} \rightarrow+\infty}\left\|x_{n_{i}}+\frac{1}{n_{i}} x_{0}\right\|=0 .
$$

Thus

$$
\lim _{n_{i} \rightarrow+\infty}\left\|B_{n_{i}} x_{n_{i}}\right\|=\lim _{n_{i} \rightarrow+\infty}\left\|B\left(x_{n_{i}}+\frac{1}{n_{i}} x_{0}\right)\right\|=+\infty .
$$


The compactness of $A$ guarantees that $\left\{A x_{n_{i}}\right\}$ has a convergent subsequence. Without loss of generality, we assume that $\lim _{n_{i} \rightarrow+\infty} A x_{n_{i}}=y_{0}$. From (2.10), we have

$$
\lim _{n_{i} \rightarrow+\infty}\left\|x_{n_{i}}\right\|=\lim _{n_{i} \rightarrow+\infty}\left\|A x_{n_{i}}+\lambda B_{n_{i}} x_{n_{i}}\right\| \geq \lim _{n_{i} \rightarrow+\infty} \lambda\left\|B_{n_{i}} x_{n_{i}}\right\|-\lim _{n_{i} \rightarrow+\infty}\left\|A x_{n_{i}}\right\|=+\infty,
$$

which contradicts (2.9).

Now (2.8) guarantees that

$$
0<\inf _{n \in \mathbb{N}}\left\|x_{n}+\frac{1}{n} x_{0}\right\| \leq\left\|x_{n}+\frac{1}{n} x_{0}\right\| \leq \sup _{x \in P \cap \Omega}\|x\|+\left\|x_{0}\right\|<+\infty, \quad n \in \mathbb{N} .
$$

Then $\left\{A x_{n}+\lambda B_{n} x_{n}\right\}$ has a convergent subsequence. Without loss of generality, we assume that

$$
A x_{n}+\lambda B_{n} x_{n} \rightarrow y_{1}, \quad \text { as } n \rightarrow+\infty .
$$

Then

$$
x_{n} \rightarrow y_{1}, \quad \text { as } n \rightarrow+\infty .
$$

Now (2.8) guarantees that $y_{1} \neq \theta$. Letting $n \rightarrow+\infty$ in (2.7), and we have

$$
y_{1}=A y_{1}+\lambda B y_{1}
$$

and $y_{1} \in P \cap \Omega-\{\theta\}$. The proof is complete.

Corollary 2.1 Suppose that $\theta \in \Omega, A: P \cap \bar{\Omega} \rightarrow P$ is continuous and compact and $B$ : $P-\{\theta\} \rightarrow P$ is singular continuous and compact. Assume that

$$
\|A x\|<\|x\|, \quad \forall x \in P \cap \partial \Omega
$$

or

$$
A x \nsupseteq x, \quad \forall x \in P \cap \partial \Omega .
$$

Then there exists $a \lambda_{*}>0$ such that, for any $\lambda \in\left(0, \lambda_{*}\right)$, there exist $x_{\lambda} \in P \cap \Omega$ with

$$
x_{\lambda}=A x_{\lambda}+\lambda B x_{\lambda} .
$$

It is easy to see that (2.12) or (2.13) guarantees that (2.1) holds (see [8]).

Theorem 2.4 Suppose that $\Omega_{1}, \Omega_{2}$ are bounded open sets and $\theta \in \Omega_{1} \subseteq \Omega_{2}, A, K: P \cap$ $\bar{\Omega}_{2} \rightarrow P$ are continuous and compact and $B: P-\{\theta\} \rightarrow P$ is singular continuous and compact. Assume that

$\left(\mathrm{C}_{1}\right) \quad A x \neq \mu x, \forall x \in P \cap \partial \Omega_{1}, \mu \geq 1$;

$\left(\mathrm{C}_{2}\right) \inf _{x \in P \cap \partial \Omega_{2}}\|K x\|>0$;

(C) $x-A x \neq \mu K x, \forall x \in P \cap \partial \Omega_{2}, \mu \geq 0$. 
Then there exists a $\lambda_{*}>0$ such that, for any $\lambda \in\left(0, \lambda_{*}\right)$, there exist $x_{\lambda, 1} \in\left(P \cap \Omega_{1}-\{\theta\}\right)$ and $x_{\lambda, 2} \in P \cap\left(\Omega_{2}-\bar{\Omega}_{1}\right)$ with

$$
x_{\lambda, 1}=A x_{\lambda, 1}+\lambda B x_{\lambda, 1}, \quad x_{\lambda, 2}=A x_{\lambda, 2}+\lambda B x_{\lambda, 2} .
$$

Proof Choose $x_{0} \in P-\{\theta\}$, and define

$$
B_{n} x=B\left(x+\frac{1}{n} x_{0}\right), \quad \forall x \in P, n \in \mathbb{N} .
$$

Set

$$
\gamma_{1}:=\inf _{(\mu, x) \in[1,+\infty) \times P \cap \partial \Omega_{1}}\|\mu x-A x\|
$$

and

$$
\gamma_{2}:=\inf _{(\mu, x) \in[0,+\infty) \times P \cap \partial \Omega_{2}}\|x-A x-\mu K x\| .
$$

We claim that

$$
\gamma_{1}>0, \quad \gamma_{2}>0
$$

An argument similar to that in (2.2) shows that

$$
\gamma_{1}>0
$$

Now we show that

$$
\gamma_{2}>0
$$

To see this suppose that $\gamma_{2}=0$. Then there exists $\left\{\left(\mu_{n}, x_{n}\right)\right\} \subseteq[0,+\infty) \times\left(P \cap \partial \Omega_{2}\right)$ such that

$$
\lim _{n \rightarrow+\infty}\left\|x_{n}-A x_{n}-\mu_{n} K x\right\|=0 .
$$

Now since

$$
\left\|x_{n}-A x_{n}-\mu_{n} K x\right\| \geq \mu_{n} \inf _{x \in \partial \cap \Omega_{2}}\|K x\|-\left\|x_{n}-A x_{n}\right\|,
$$

we have $\left\{\mu_{n}\right\}$ is bounded, which means that $\left\{\mu_{n}\right\}$ has a convergent subsequence. Without loss of generality, we assume that

$$
\lim _{n \rightarrow+\infty} \mu_{n}=\mu_{0}
$$

Since $\left\{x_{n}\right\}$ is bounded and $A$ and $K$ are compact, $\left\{A x_{n}\right\}$ and $\left\{K x_{n}\right\}$ have convergent subsequences $\left\{A x_{n_{i}}\right\}$ and $\left\{K x_{n_{i}}\right\}$ with $\lim _{n_{i} \rightarrow+\infty} A x_{n_{i}}=y_{0}$ and $\lim _{n_{i} \rightarrow+\infty} K x_{n_{i}}=z_{0}$. Now

$$
\lim _{n \rightarrow+\infty}\left\|x_{n}-A x_{n}-\mu_{n} K x_{n}\right\|=0
$$


which implies that

$$
\lim _{n_{i} \rightarrow+\infty}\left\|x_{n_{i}}-y_{0}-\mu_{0} z_{0}\right\|=0
$$

Let $x_{0}=y_{0}+\mu_{0} z_{0}$. Clearly, $x_{0} \in P \cap \partial \Omega_{2}$. Now

$$
\left\|x_{0}-A x_{0}-\mu_{0} K x_{0}\right\|=\lim _{n_{i} \rightarrow+\infty}\left\|x_{n_{i}}-A x_{n_{i}}-\mu_{n_{i}} K x_{n_{i}}\right\|=0
$$

which contradicts condition $\left(\mathrm{C}_{3}\right)$.

Consequently, (2.16) is true, which together with (2.15) yields (2.14).

Let $\gamma=\min \left\{\gamma_{1}, \gamma_{2}\right\}$. Obviously, $\gamma>0$.

Let

$$
\beta_{n, 1}=\sup _{x \in P \cap \partial \Omega_{1}}\left\|B_{n} x\right\|, \quad \beta_{n, 2}=\sup _{x \in P \cap \partial \Omega_{2}}\left\|B_{n} x\right\| .
$$

An argument similar to that in (2.4) shows that

$$
\sup _{n \in \mathbb{N}} \beta_{n, 1}<+\infty, \quad \sup _{n \in \mathbb{N}} \beta_{n, 2}<+\infty .
$$

Let

$$
\beta:=\max \left\{\sup _{n \in \mathbb{N}} \beta_{n, 1}, \sup _{n \in \mathbb{N}} \beta_{n, 1}\right\}<+\infty
$$

and

$$
\lambda_{*}:=\frac{\gamma}{\beta}>0
$$

For $0<\lambda<\lambda_{*}, x \in P \cap \partial \Omega_{1}, \mu \geq 1$, we have

$$
\left\|A x+\lambda B_{n} x-\mu x\right\| \geq\|A x-\mu x\|-\lambda\left\|B_{n} x\right\| \geq \gamma-\beta \lambda>0,
$$

which guarantees that

$$
i\left(A+\lambda B_{n}, P \cap \Omega_{1}, P\right)=1, \quad n \in \mathbb{N},
$$

and for $x \in P \cap \partial \Omega_{2}, \mu \geq 0$, we have

$$
\left\|x-\left(A x+\lambda B_{n} x\right)-\mu K x\right\| \geq\|x-A x-\mu K x\|-\lambda\left\|B_{n} x\right\| \geq \gamma-\beta \lambda>0,
$$

which guarantees that

$$
i\left(A+\lambda B_{n}, P \cap \Omega_{2}, P\right)=0 .
$$

Thus

$$
i\left(A+\lambda B_{n}, P \cap\left(\Omega_{2}-\bar{\Omega}_{1}\right), P\right)=-1 .
$$


Now (2.18) and (2.19) guarantee that there exist $\left\{x_{n, 1}\right\} \subseteq P \cap \Omega_{1}$ and $\left\{x_{n, 2}\right\} \subseteq P \cap\left(\Omega_{2}-\bar{\Omega}_{1}\right)$ such that

$$
x_{n, 1}=A x_{n, 1}+\lambda B_{n} x_{n, 1}, \quad x_{n, 2}=A x_{n, 2}+\lambda B_{n} x_{n, 2}, \quad \lambda \in\left(0, \lambda_{*}\right), n \in \mathbb{N} .
$$

An argument similar to that in (2.11) shows that there exist $y_{1} \in P \cap \Omega_{1}-\{\theta\}$ and $y_{2} \in$ $P \cap\left(\Omega_{2}-\bar{\Omega}_{1}\right)$ with

$$
y_{1}=A y_{1}+\lambda B y_{1}, \quad y_{2}=A y_{2}+\lambda B y_{2}, \quad \lambda \in\left(0, \lambda_{*}\right) .
$$

The proof is complete.

Corollary 2.2 Suppose that $\Omega_{1}, \Omega_{2}$ are bounded open sets and $\theta \in \Omega_{1} \subseteq \Omega_{2}, A: P \cap \bar{\Omega}_{2} \rightarrow$ $P$ is continuous and compact and $B: P-\{\theta\} \rightarrow P$ is singular continuous and compact. Assume that

$\left(\mathrm{C}_{4}\right)$

$$
\|A x\|<\|x\|, \quad \forall x \in P \cap \partial \Omega_{1}
$$

or

$$
A x \nsupseteq x, \quad \forall x \in P \cap \partial \Omega_{1} ;
$$

$\left(C_{5}\right)$

$$
\begin{gathered}
\|A x\|>\|x\|, \quad \forall x \in P \cap \partial \Omega_{2} \\
\text { or } \exists u_{0} \in P-\{\theta\} \text { such that } \\
x-A x \neq \mu u_{0}, \quad \forall x \in P \cap \partial \Omega_{2}, \mu \geq 0
\end{gathered}
$$

or

$$
A x \not \leq x, \quad \forall x \in P \cap \partial \Omega_{2} .
$$

Then there exists a $\lambda_{*}>0$ such that, for any $\lambda \in\left(0, \lambda_{*}\right)$, there exist $x_{\lambda, 1} \in P \cap \Omega_{1}-\{\theta\}$ and $x_{\lambda, 2} \in P \cap\left(\Omega_{2}-\bar{\Omega}_{1}\right)$ with

$$
x_{\lambda, 1}=A x_{\lambda, 1}+\lambda B x_{\lambda, 1}
$$

and

$$
x_{\lambda, 2}=A x_{\lambda, 2}+\lambda B x_{\lambda, 2} .
$$

It is easy to see that $\left(C_{4}\right)$ and $\left(C_{5}\right)$ guarantee that $\left(C_{1}\right)-\left(C_{3}\right)$ hold (see [8]). 


\section{Applications for singular systems of multi-point boundary value problems}

In [9], Henderson and Luca considered the system of nonlinear second-order ordinary differential equations

$$
\begin{cases}u^{\prime \prime}(t)+f(t, v(t))=0, & t \in(0, T), \\ v^{\prime \prime}(t)+g(t, u(t))=0, & t \in(0, T)\end{cases}
$$

with multi-point boundary conditions

$$
\begin{cases}u(0)=0, & u(T)=\sum_{i=1}^{m-2} b_{i} u\left(\xi_{i}\right), \quad m \geq 3 \\ v(0)=0, & v(T)=\sum_{i=1}^{n-2} c_{i} v\left(\eta_{i}\right), \quad n \geq 3\end{cases}
$$

The following conditions come from [9]:

(H1) $0<\xi_{1}<\cdots<\xi_{m-2}<T, 0<\eta_{1}<\cdots<\eta_{n-2}<T, b_{i}>0, i=1,2, \ldots, m-2, c_{i} \geq 0$, $i=1,2, \ldots, n-2, d=T-\sum_{i=1}^{m-2} b_{i} \xi_{i}>0, e=T-\sum_{i=1}^{n-2} c_{i} \eta_{i}>0, \sum_{i=1}^{m-2} b_{i} \xi_{i}>0$, $\sum_{i=1}^{n-2} c_{i} \eta_{i}>0$,

(H2) we have the functions $f, g \in C([0, T] \times[0,+\infty),[0,+\infty))$ and $f(t, 0)=0, g(t, 0)=0$ for all $t \in[0, T]$,

(H3) there exists a positive constant $p \in(0,1]$ such that

(1) $f_{\infty}^{i}=\lim _{u \rightarrow+\infty} \operatorname{infinf}_{t \in[0, T]} \frac{f(t, u)}{u^{p}} \in(0,+\infty]$;

(2) $g_{\infty}^{i}=\lim _{u \rightarrow+\infty} \operatorname{infinf}_{t \in[0, T]} \frac{g(t, u)}{u^{\frac{1}{p}}}=+\infty$,

(H4) there exists a $r \in(0,+\infty)$ such that

(1) $f_{\infty}^{s}=\lim _{u \rightarrow+\infty} \sup \sup _{t \in[0, T]} \frac{f(t, u)}{u^{r}} \in(0,+\infty]$;

(2) $g_{\infty}^{s}=\lim _{u \rightarrow+\infty} \sup \sup _{t \in[0, T]} \frac{g(t, u)}{u^{\frac{1}{r}}}=0$,

(1) $f_{0}^{i}=\lim _{u \rightarrow 0+} \operatorname{infinf}_{t \in[0, T]} \frac{f(t, u)}{u} \in(0,+\infty]$;

(2) $g_{0}^{i}=\lim _{u \rightarrow 0+} \operatorname{infinf}_{t \in[0, T]} \frac{g(t, u)}{u}=+\infty$,

(H6) for each $t \in[0, T], f(t, u)$ and $g(t, u)$ are nondecreasing with respect to $u$, and there exists a constant $N>0$ such that

$$
f\left(t, m_{0} \int_{0}^{T} g(s, N) d s\right)<\frac{N}{m_{0}}, \quad \forall t \in[0, T],
$$

where $m_{0}=\frac{T^{2}}{4} \max \left\{a_{1} T, \tilde{a}_{1}\right\}$ and $a_{1}, \tilde{a}_{1}$ are defined in [9].

Theorem 3.1 ([9]) Assume that (H1)-(H2) and (H4)-(H5) hold. Then Problem (3.1), (3.2) has at least one positive solution $(u(t), v(t)), t \in[0, T]$.

Theorem 3.2 ([9]) Assume that (H1)-(H3) and (H5)-(H6) hold. Then Problem (3.1), (3.2) has at least two positive solutions $\left(u_{1}(t), v_{1}(t)\right),\left(u_{2}(t), v_{2}(t)\right), t \in[0, T]$.

Here we consider

$$
\left\{\begin{array}{l}
u^{\prime \prime}(t)+f(t, v(t))+\lambda u^{-\gamma}=0, \quad t \in(0, T) \\
v^{\prime \prime}(t)+g(t, u(t))=0, \quad t \in(0, T)
\end{array}\right.
$$

with multi-point boundary conditions (3.2), where $1>\gamma>0$. 
Let $C[0, T]:=\{x:[0, T] \rightarrow R: x(t)$ is continuous on $[0, T]\}$ with norm

$$
\|x\|=\max _{t \in[0, T]}|x(t)| .
$$

Obviously, $C[0, T]$ is a Banach space. Let

$$
P:=\left\{x \in C[0, T]: x(t) \geq 0 \text { is concave and } \inf _{t \in\left[\theta_{0}, T\right]} x(t) \geq \gamma\|x\|\right\},
$$

where $\theta_{0}$ and $\gamma$ are defined in Section 2 in [9].

For $u \in P$, define an operator

$$
(A u)(t)=\int_{0}^{T} G_{1}(t, s) f\left(s, \int_{0}^{T} G_{2}(s, \tau) g(\tau, u(\tau)) d \tau\right) d s, \quad t \in[0, T]
$$

and for $u \in P-\{\theta\}$, define an operator

$$
(B u)(t)=\int_{0}^{T} G_{1}(t, s) u^{-\gamma}(s) d s, \quad t \in[0, T],
$$

where $G_{1}(t, s)$ and $G_{2}(t, s)$ are defined in [9].

It is easy to see that $B: P-\{\theta\} \rightarrow P$ is a singular continuous and compact operator (see $[9,11])$.

Theorem 3.3 Assume that $(\mathrm{H} 1)-(\mathrm{H} 2)$ and $(\mathrm{H} 4)$ hold. Then there exists a $\lambda^{*}>0$ such that Problem (3.3), (3.2) has at least one positive solution $(u(t), v(t)), t \in[0, T]$ for all $\lambda \in\left(0, \lambda^{*}\right)$.

Proof Let $B_{R}$ be defined as that in Theorem 3.2 of [9]. From the proof in [9], it is easy to see that

$$
\|A x\|<\|x\|, \quad \forall x \in P \cap \partial B_{R} .
$$

Now Corollary 2.1 guarantees that there exists a $\lambda^{*}>0$ such that, for any $\lambda \in\left(0, \lambda^{*}\right)$, there exist $u \in\left(P \cap B_{R}-\{\theta\}\right)$ such that

$$
u=A u+\lambda B u
$$

Let

$$
v(t)=\int_{0}^{T} G_{2}(t, s) g(s, u(s)) d s, \quad t \in[0, T] .
$$

Then $(u(t), v(t))$ is a positive solution for (3.3), (3.2). The proof is complete.

Theorem 3.4 Assume that (H1)-(H3) and (H6) hold. Then Problem (3.3), (3.2) has at least two positive solutions $\left(u_{1}(t), v_{1}(t)\right),\left(u_{2}(t), v_{2}(t)\right), t \in[0, T]$.

Proof Let $B_{N}$ and $B_{L}$ be defined as in Theorem 3.3 of [9]. From the proof in [9], it is easy to see that

$$
\begin{aligned}
& \|A x\|<\|x\|, \quad \forall x \in P \cap \partial B_{N}, \\
& x \neq A x+\lambda u_{0}, \quad \forall x \in P \cap \partial B_{L}, \lambda \geq 0, L>N .
\end{aligned}
$$


Now Corollary 2.2 guarantees that there exists a $\lambda^{*}>0$, for any $\lambda \in\left(0, \lambda^{*}\right)$, such that there exist $u_{1} \in\left(P \cap B_{N}-\{\theta\}\right)$ and $u_{2} \in P \cap\left(B_{L}-\bar{B}_{N}\right)$ with

$$
u_{1}=A u_{1}+\lambda B u_{1}
$$

and

$$
u_{2}=A u_{2}+\lambda B u_{2}
$$

Let

$$
v_{1}(t)=\int_{0}^{T} G_{2}(t, s) g\left(s, u_{1}(s)\right) d s, \quad t \in[0, T]
$$

and

$$
\nu_{2}(t)=\int_{0}^{T} G_{2}(t, s) g\left(s, u_{2}(s)\right) d s, \quad t \in[0, T] .
$$

Then $\left(u_{1}(t), v_{1}(t)\right)$ and $\left(u_{2}(t), v_{2}(t)\right)$ are two positive solutions for (3.3), (3.2). The proof is complete.

Remark Note that $f$ and $g$ have no singularity at $u=0$ and $v=0$ in $[9,10]$, so Theorems 3.3 and 3.4 improve the results in $[9,10]$.

\section{Competing interests}

The authors declare that they have no competing interests.

\section{Authors' contributions}

All authors contributed equally to the paper. All authors read and approved the final manuscript.

\section{Author details}

${ }^{1}$ Department of Mathematics, Shandong Normal University, Ji-nan, 250014, China. ${ }^{2}$ School of Mathematics, Statistics and Applied Mathematics, National University of Ireland, Galway, Ireland. ${ }^{3}$ Department of Mathematics, Faculty of Science, King Abdulaziz University, Jeddah, Saudi Arabia. ${ }^{4}$ Department of Mathematics, Texas A\&M University-Kingsville, Kingsville, Texas 78363, USA.

\section{Acknowledgements}

This research is supported by Young Award of Shandong Province (ZR2013AQ008).

Received: 11 November 2013 Accepted: 3 January 2014 Published: 24 Jan 2014

\section{References}

1. Aris, R: The Mathematical Theory of Diffusion and Reaction of Permeable Catalysts. Clarendon, Oxford (1975)

2. Callegari, A, Nachman, A: Some singular nonlinear differential equations arising in boundary layer theory. J. Math. Anal. Appl. 64, 96-105 (1978)

3. Callegari, A, Nachman, A: A nonlinear singular boundary value problem in the theory of pseudoplastic fluids. SIAM J. Appl. Math. 38, 275-282 (1980)

4. Taliaferro, S: A nonlinear singular boundary value problem. Nonlinear Anal. 3, 897-904 (1979)

5. Agarwal, RP, O'Regan, D: A survey of recent results for initial and boundary value problems singular in the dependent variable. In: Handbook of Differential Equations: Ordinary Differential Equations, vol. 1, pp. 1-68 (2000)

6. Agarwal, RP, O'Regan, D: Existence theory for single and multiple solutions to singular positone boundary value problems. J. Differ. Equ. 175, 393-414 (2001)

7. Ge, W: Boundary Value Problems for Nonlinear Ordinary Differential Equations. Science Press, Beijing (2007) (in Chinese)

8. Guo, D, Lakshmikantham, V: Nonlinear Problems in Abstract Cones. Academic Press, New York (1988)

9. Henderson, J, Luca, R: Existence and multiplicity for positive solutions of a multi-point boundary value problems. Appl. Math. Comput. 218, 10572-10585 (2012) 
10. Henderson, J, Luca, R: Positive solutions for singular multi-point boundary value problems. Math. Methods Appl. Sci. 36, 814-828 (2013)

11. Liu, B, Liu, L, Wu, Y: Positive solutions for singular second order three-point boundary value problems. Nonlinear Anal., Theory Methods Appl. 66, 2756-2766 (2007)

12. Ma, R: Nonlocal Problems for Nonlinear Ordinary Differential Equations. Science Press, Beijing (2004) (in Chinese)

13. O'Regan, D: Existence Theory for Ordinary Differential Equations. Kluwer Academic, Dordrecht (1997)

14. O'Regan, D: Theory of Singular Boundary Value Problems. World Scientific, Singapore (1994)

10.1186/1029-242X-2014-37

Cite this article as: Yan et al.: Fixed-point theorems for nonlinear operators with singular perturbations and applications. Journal of Inequalities and Applications 2014, 2014:37

Submit your manuscript to a SpringerOpen ${ }^{\circ}$ journal and benefit from:

- Convenient online submission

- Rigorous peer review

- Immediate publication on acceptance

Open access: articles freely available online

- High visibility within the field

- Retaining the copyright to your article 